\title{
Nonreciprocal propagation in a non-Hermitian silicon photonic coupler employing graphene saturable absorption
}

\author{
Dimitrios Chatzidimitriou ${ }^{1, *}$, Alexandros Pitilakis ${ }^{1,}$, Traianos Yioultsis ${ }^{1,}$, and Emmanouil Kriezis ${ }^{1,}$ \\ ${ }^{1}$ Department of Electrical and Computer Engineering, Aristotle University of Thessaloniki, 54124 Thessaloniki, Greece
}

\begin{abstract}
We present the concept, analysis and design of a nonreciprocal system of coupled nonlinear waveguides, operating at the near infrared. We exploit the spatial asymmetry induced by the exceptional points of a non-Hermitian coupler which, combined with saturable absorption in the nonlinear regime, can break Lorentz reciprocity. In this work, we introduce asymmetric losses in a pair of coupled silicon waveguides by overlaying only one of them with unbiased graphene, subject to saturable absorption. The device can be used as a broadband half-duplex isolator: a two-port component offering unidirectional transmission of high-power signals.
\end{abstract}

\section{Introduction}

Components with nonreciprocal response are highly desirable in integrated photonics. Diodes, the elementary nonreciprocal components, exhibit unidirectional transmission and are the building blocks of advanced components such as isolators, circulators, and logic gates; these components are of fundamental importance in even more complex devices such as sources (lasers), tranceivers, and all-optical processing units. Presently, magnetic materials exhibiting Faraday rotation are mostly used for optical isolators, but they are bulky and costly to hybridly integrate in photonic platforms. Consequently, alternative ways have been proposed to break Lorentz reciprocity, such as the combination of nonlinearity and spatial asymmetry. This concept has been mainly explored using the Kerr effect in photonic resonators.

In this work, we present a concept broadband photonic diode relying on graphene saturable absorption (SA) in an asymmetric coupler. In the near infrared and for a chemical potential $\left(\mu_{c}\right)$ below the half photon energy, graphene's absorption is predominately due to interband transitions. This absorption is broadband, saturable, i.e., decreases with increasing intensity, has a sub-ps response time, and a much lower power threshold compared to the Kerr effect. Our concept is based on the existence of exceptional points (EPs) [1], singularities in the eigenvalue space of non-Hermitian systems. In our implementation, the EP in a lossy waveguide coupler combined with the asymmetric field overlap with the nonlinear SA material gives rise to nonreciprocal functionality, manifested as uni-directional transmission.

\section{Concept, analysis and design}

We consider the abstract waveguide coupler depicted in Fig. 1(a), consisting of one lossless waveguide (blue) and

*e-mail: dcthazid@ece.auth.gr one nonlinear lossy waveguide (orange) whose losses are fully saturable. The waveguides are otherwise identical, i.e., their individual modes have the same propagation constant (real part). The coupler is configured as a two-port component with ports 1 and 2 denoting opposite ends of the lossy and lossless waveguides, respectively.

Assuming that the coupler's length is equal to the coupling length $L \approx L_{c}=\pi / 2 \kappa$, where $\kappa$ is the coupling coefficient, then for low power input the response of the device is dictated by the operation above the EP: Guided power in the lossless waveguide is weakly coupled to the lossy one, while power guided in the lossy waveguide is greatly attenuated before being weakly coupled to the lossless waveguide [2]. For the port configuration in Fig. 1(a) this translates to very low reciprocal transmission. For high power excitation from the lossy waveguide (port 1), losses are saturated allowing full coupling to the opposite waveguide. Thus, transmission to port 2 will be high; this is the through direction. On the contrary, excitation of the lossless waveguide does not sufficiently overlap with the SA material and coupling is suppressed due to the EP, leading to low transmission to port 1 ; this is the isolated direction.

A coupled mode framework can be developed for the qualitative analysis and design of the device. Assuming that $z$ is the propagation direction, $A_{1 / 2}$ is the E-field complex amplitude on the top/bottom waveguide normalized by the saturation amplitude $\left(\left|A_{\text {sat }}\right|\right), \beta$ is the real phase constant for both waveguides, and $\alpha$ is the low-power attenuation coefficient for the lossy waveguide, then the coupled equation system describing the device is

$$
\frac{\partial}{\partial z}\left[\begin{array}{l}
A_{1} \\
A_{2}
\end{array}\right]=\left[\begin{array}{cc}
-j \beta-\frac{\alpha}{1+\left|A_{1}\right|^{2}} & \kappa \\
-\kappa^{*} & -j \beta
\end{array}\right]\left[\begin{array}{l}
A_{1} \\
A_{2}
\end{array}\right] .
$$

Solving this system for an initial excitation at one port leads to the transmission coefficients in both directions, the forward $T_{21}$ and backward $T_{12}$. Note that we assume excitation of only one port at any time. In the linear regime, 


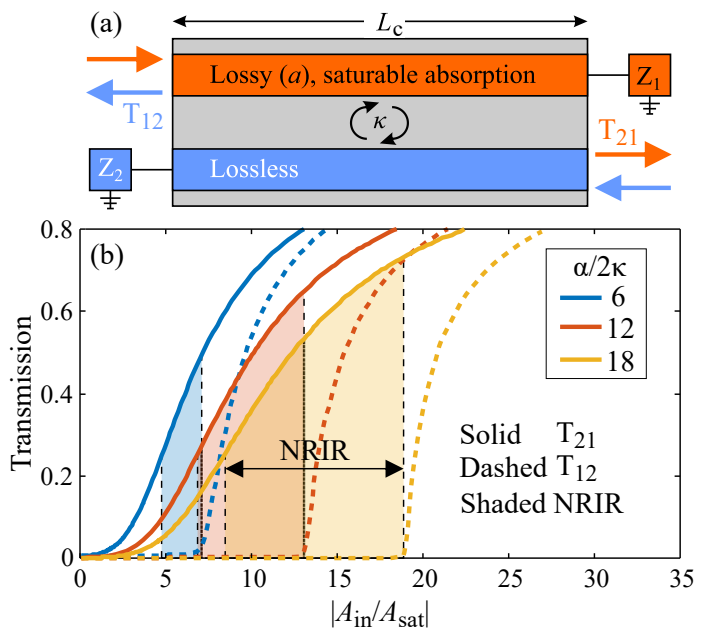

Figure 1. (a) Abstract two-port nonlinear asymmetric waveguide coupler of length $L=L_{c}=\pi / 2 \kappa$; terminals $Z_{1,2}$ denote matched loads. (b) Forward $\left(T_{21}\right)$ and backward $\left(T_{12}\right)$ transmission vs. normalized input amplitude; colors correspond to different values of the loss parameter $\alpha / 2 \kappa$.

the EP is located at $\alpha / 2 \kappa=1$. In the nonlinear regime, the state of the system is power dependent, i.e., high power signals alter the state relative to the EP, which drastically changes the response. The transmission coefficients for $\alpha / 2 \kappa=6,12$ and 18 vs. the normalized input field amplitude are presented in Fig. 1(b), where the shaded regions corresponds to $T_{21} \geq-6 \mathrm{~dB}$ and $T_{12} \leq-15 \mathrm{~dB}$; this is the Non-Reciprocal Intensity Range (NRIR) of this device [3]. Note that low power signals are heavily attenuated in both directions and also there exists a limiting power threshold beyond which isolation abruptly drops ( $T_{12}$ rises) and the device is transparent in both directions. The latter happens when even the low fraction of power coupled from the lossless to the lossy waveguide is able to sufficiently saturate losses to restore normal coupling operation.

The physical implementation of the coupler is shown in the inset of Fig. 2. Each Si-slot waveguide consists of two identical ribs, $360 \mathrm{~nm}$ wide and $180 \mathrm{~nm}$ tall, forming a $40 \mathrm{~nm}$ slot, while the gap is $640 \mathrm{~nm}$ leading to $L_{c}=800 \mu \mathrm{m}$. On the top face of the left waveguide two uncoupled graphene layers are deposited, which are assumed to be unbiased $\left(\mu_{c}=0 \mathrm{eV}\right)$ and possess a saturation intensity of $I_{\mathrm{sat}}=1 \mathrm{MW} / \mathrm{cm}^{2}$. The two layers have a total surface conductivity of $121.7 \mu \mathrm{S}$, leading to $0.42 \mathrm{~dB} / \mu \mathrm{m}$ losses, assumed fully saturable.

For the validation, we firstly derive a pair of nonlinear Schrödinger equations (NLSE) [4], for the TE mode of each isolated waveguide, heuristically coupled by the weak coefficient $\kappa=\pi / 2 L_{c}$. The SA coefficient of the graphene-overlaid waveguide is formally introduced by using the overlap of the mode distribution with graphene. The simulation results are shown in Fig. 2. Although there are moderate forward losses (6 to $3 \mathrm{~dB})$, the threshold CW power for nonreciprocal regime is very low (160 to $320 \mathrm{~mW}$ ), which showcases graphene's low saturation intensity. Note that the bandwidth limiting factor is the wavelength dispersion of the coupling coefficient and mode profile, both of which are low. The results were validated by full-vector 3D beam propagation method (BPM) simulations, depicted with markers in Fig. 2; the custom BPM was implemented with finite elements in the cross-section and wide-angle Crank-Nicolson scheme in the propagation direction, iteratively stabilized for nonlinearity.

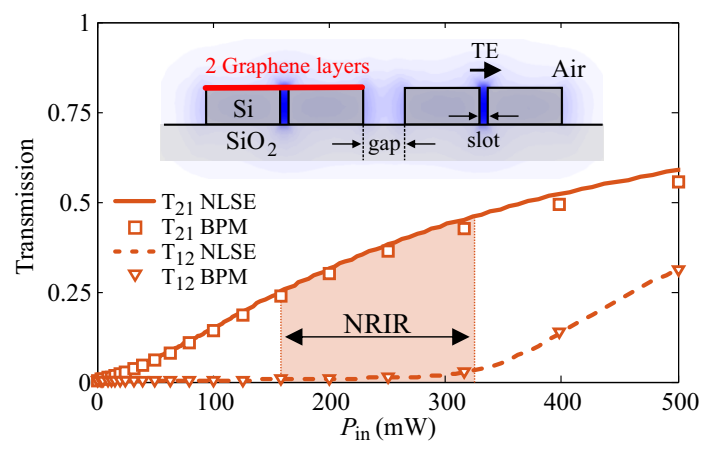

Figure 2. Forward (solid) and backward (dashed) transmission for the graphene waveguide coupler with SA. Shaded region corresponds to $T_{21} \geq-6 \mathrm{~dB}$ and $T_{12} \leq-15 \mathrm{~dB}$. (Inset) Cross section (not to scale) of the silicon slot coupler with the symmetric supermode. The arrow indicates the dominant mode polarization.

\section{Conclusions}

Our analysis, design and evaluation show that asymmetric coupled graphene-overlaid waveguides can operated as nonlinear optical diodes, compatible with standard photonic integrated circuit technology. Our approach towards nonreciprocity provides an alternative route to the more commonly used Kerr effect, thanks to graphene's SA ultra fast response and low saturation intensity. Finally, our concept has a general scope and demonstrates how SA in conjunction with EPs can be exploited to design nonreciprocal integrated photonic components.

\section{Acknowledgment}

This research is co-financed by Greece and the European Union (European Social Fund- ESF) through the Operational Programme «Human Resources Development, Education and Lifelong Learning 2014-2020» in the context of the project "Design of nonlinear silicon devices incorporating graphene and using the Parity time symmetry concept" (MIS 5047874).

\section{References}

[1] M.A. Miri, A. Alù, Science 363 (2019)

[2] D. Chatzidimitriou, E.E. Kriezis, J. Opt. Soc. Am. B 35, 1525 (2018)

[3] D.L. Sounas, A. Alù, Physical Review B 97 (2018)

[4] D. Chatzidimitriou, A. Pitilakis, E.E. Kriezis, Journal of Applied Physics 118, 023105 (2015) 